\title{
A New Approach to Optimization of Dynamic Reactive Power Sources Addressing FIDVR Issues
}

\author{
Weihong Huang, Kai Sun, Junjian Qi \\ University of Tennessee \\ Knoxville, TN, USA \\ whuang12@utk.edu \\ kaisun@utk.edu \\ jqi1@utk.edu
}

\author{
Yan $\mathrm{Xu}$ \\ Oak Ridge National Laboratory \\ Oak Ridge, TN, USA \\ xuy3@ornl.gov
}

\begin{abstract}
Dynamic reactive power (var) sources, e.g. SVCs and STATCOMs, can effectively mitigate fault-induced delayed voltage recovery (FIDVR) issues or even voltage collapse. However, their optimal allocation in a power grid is a complicated nonlinear optimization problem since the post-fault voltage trajectories have to be solved to check constraints on voltage responses. Thus, solvers of both nonlinear optimization and power system differential and algebraic equations (DAEs) are required. This paper proposes a new approach to optimize the sizes of dynamic var sources at candidate locations by efficiently interfacing a heuristic linear programming based searching algorithm with power system simulation software. Case studies on a 9-bus system and the IEEE 39-bus system have benchmarked the new approach with an existing representative approach and demonstrated that the new approach can quickly converge to an optimal solution.
\end{abstract}

Index Terms-dynamic var support; FIDVR; linear programming, nonlinear optimization; voltage recovery

\section{INTRODUCTION}

Voltage security issues in a power grid have attracted more attention due to ever-growing electricity demands. Other than depressed voltage profiles, utilities also have increasing concerns with dynamic voltage security issues, especially fault-induced delayed voltage recovery (FIDVR) issues at load buses and even fast voltage collapse following cascading events, because of the increasing dynamic fastresponding loads, such as the air conditions with highefficiency low-inertia motors. Allocation and management of var sources, especially dynamic var sources, become an emerging research topic.

Some papers have studied the optimal allocation of dynamic var sources [1]-[6]. Reference [1] studies the placement of SVCs by using post-contingency trajectory sensitivity analysis. In [2], the sizes and locations of var sources are determined by means of optimal power flow (OPF), and verified by P-V and Q-V analysis. In [3], OPF is also applied to determine a right mix of static and dynamic var supports. Mid-term time-domain simulation is performed for refining the solution. In [4], a technique called "the time continuation method" is applied to find the locations and amounts of dynamic var sources, coupling with a quadratic model of the electric power system, including generators, voltage regulators, and induction motors. In [5] and [6], the dynamic var allocation problem is formulated as a mixed integer nonlinear programing problem. The var locations and an initial guess on var sizes are respectively from a trajectory sensitivity study and a linear programming (LP) algorithm [1], and then the final solution is obtained by interfacing an optimization tool with power system time-domain simulation software to optimize the required var amounts for each time step of the post-contingency trajectories [5].

The complexity of the optimal allocation of dynamic var sources is mainly because the problem itself is a nonlinear optimization problem and checking its constraints requires knowing post-fault power system trajectories. Thus both a nonlinear optimization solver and a power system DAE solver are required and they should be systematically integrated or interfaced for an efficient algorithm to solve the problem. Most of the existing methods either solve a simplified problem for an approximate solution or suffer computational burdens for large-scale power systems if two solvers are interacted frequently.

The approach proposed in this paper solves the optimal dynamic var allocation problem by systematically interfacing power system time-domain simulation software with a LP based heuristic search algorithm. Compared to the approach in [6], it takes a small number of iterative steps to quickly converge to an optimal solution, and at each step, the LP can effectively identify the searching direction for reducing the cost function toward an optimum. Thus, the new approach has moderate computation burdens. The proposed approach is tested on the WSCC 9-bus system and the IEEE 39-bus system.

\section{FORMULATION OF THE PROBLEM}

To address the fault-induced delayed voltage recovery (FIDVR) issues, power companies follow certain planning standards to plan dynamic var sources to limit voltage dip/sag after a fault [7]. Without losing generality, this paper

This manuscript has been authored by UT-Battelle, LLC, under Contract No. DE-AC05-00OR22725 with the U.S. Department of Energy. The United States Government retains and the publisher, by accepting the article for publication, acknowledges that the United States Government retains a nonexclusive, paid-up, irrevocable, world-wide license to publish or reproduce the published form of this manuscript, or allow others to do so, for United States Government purposes. 
considers typical voltage criteria according to the WECC/NERC planning standards [8]. For the most severe N1 or $\mathrm{N}-2$ contingency, the post-fault transient voltage performance is expected to meet criteria such as:

1. The post-fault voltage deviation at a bus relative to its pre-fault initial voltage should not exceed $\mathrm{D}_{1} \%$.

2. The duration of a post-fault voltage dip or overshoot $>\mathrm{D}_{2} \%$ should not exceed L cycles.

3. The post transient (starting from $t_{s}$ seconds) voltage deviation at a bus should not exceed $\mathrm{D}_{3} \%$.

This paper considers typical parameters in the criteria:

- $\mathrm{D}_{1}=25(\%)$ for load buses and 30(\%) for generator buses

- $\mathrm{D}_{2}=20(\%)$ and $\mathrm{L}=20$ (cycles)

- $t_{s}=3$ (seconds) after the fault clearing time (denoted by $\left.t_{c l}\right)$ and $\mathrm{D}_{3}=5(\%)$

Fig. 1 illustrates the criteria for a load bus.

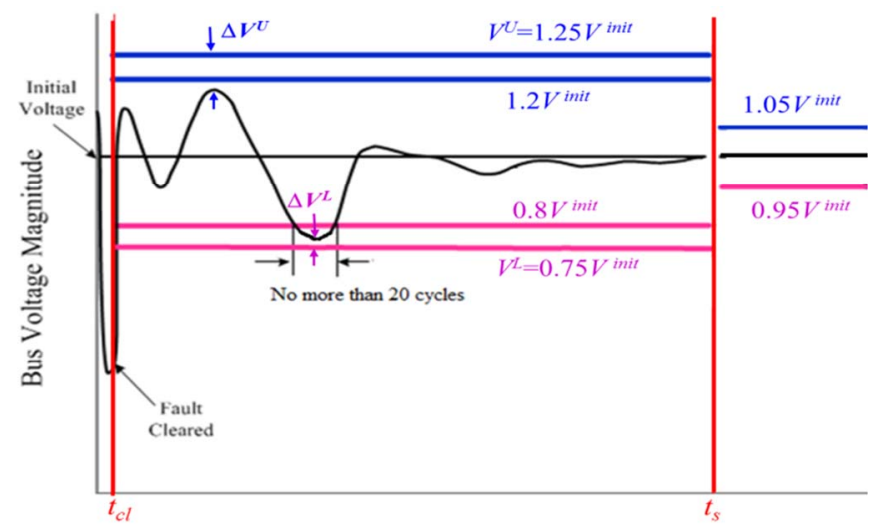

Figure 1. Post-fault voltage performance criteria

Assume the system has $N$ buses. Define the percentage voltage deviation of a bus $j$ at time $t$ :

$$
R_{j}(t)=\left|\frac{V_{j}(t)-V_{j}^{\text {init }}}{V_{j}^{\text {init }}}\right| \times 100 \% \quad j=1 \sim N
$$

where $V_{j}^{i n i t}$ is the pre-fault initial voltage magnitude. Thus, the optimal allocation problem for dynamic var sources (taking SVC as an example) can be formulated as follows. Minimize

$$
f=\sum_{i \in I_{Q}} c_{i} Q_{i}
$$

Subject to

$$
\begin{gathered}
Q_{i}^{L} \leq Q_{i} \leq Q_{i}^{U} \quad \forall i \in I_{Q} \\
R_{j}(t) \leq 25 \%, \quad t_{c l} \leq t<t_{s}, \forall j \in I_{L} \\
R_{j}(t) \leq 30 \%, \quad t_{c l} \leq t<t_{s}, \forall j \in I_{G} \\
R_{j}(t) \leq 5 \%, \quad t \geq t_{s}, \forall j=1 \sim N \\
\text { Duration }_{R_{j}(t) \geq 20 \%}<20 \text { cycles, } \quad t_{c l} \leq t<t_{s}, \forall j=1 \sim N \\
\dot{x}=f(x, y, Q)
\end{gathered}
$$

where $Q_{i}$ is the dynamic var injected at candidate bus $i, c_{i}$ is the cost coefficient associated with $Q_{i}$, e.g. the cost per Mvar of a SVC, $Q_{i}{ }^{L}$ and $Q_{i}{ }^{U}$ are the lower and upper limits of $Q_{i}, I_{Q}$ is the set of candidate buses, $I_{L}$ and $I_{G}$ are respectively the sets of load and generator buses, equation (8) represents the differential and algebraic equations (DAEs) on power system dynamics, $x$ and $y$ are vectors of differential variables and algebraic variables, and $Q$ is the vector of $Q_{i}$.

With the post-fault voltage trajectories from time-domain simulation, the above constraint (7) may be checked for each simulation time step $\Delta t$. For example, if $\Delta t=0.005 \mathrm{~s}, 20$ cycles in $60 \mathrm{~Hz}$ systems correspond to about 67 time steps. Then, that constraint requires that a $>20 \%$ voltage deviation should not last for more than 67 steps.

The region of all solutions for the above optimization problem (2) (8) may be non-convex, and that optimization is coupled with solving DAEs for post-fault power system trajectories. Therefore, it would be very complicated to directly solve the problem. For example, for an exhaustive searching method, it would need to solve the post-fault voltage response with any potential combination of values of $Q_{i}$ 's and then check voltage criteria so as to judge whether it is a feasible solution or not. The paper proposes an efficient approach to an optimal solution of the problem.

\section{PROPOSED APPROACH}

\section{A. Determine the Locations of Dynamic Var Sources}

For the most severe contingency, some candidate buses (i.e. $I_{Q}$ ) are selected among all $N$ buses in the system. The candidate buses may be those where var injection will cause more sensitive improvements on the overall post-fault voltage level. For instance, the method based on a post-fault trajectory sensitivity index in [1] may be applied. Another similar index is the Voltage Sensitivity Index (VSI) defined below. A small amount of dynamic var $q_{i}$ is assumed to be injected at bus $i$, which can be simulated by adding a SVC of size $q_{i}$. Assume that the simulation time step is $\Delta t$, the total simulation period is $T \times \Delta t$, and the var injection changes the voltage at bus $j$ from $V_{j}^{\text {old,t }}$ to $V_{j}^{\text {new, }}$ at time step $t$ from time-domain simulation results. VSI is calculated by (9) for each bus $j$ over the simulation period, which equals the maximum voltage recovery sensitivity $(>0)$ at all the time steps.

$$
V S I_{i j}=\max \left[V_{j}^{\text {new }, t}-V_{j}^{\text {old }, t}, t=1 \sim T\right] / q_{i}
$$

Then the average recovery sensitivity for the system about the var injection at bus $i$ is

$$
V S I_{i}=\frac{1}{N} \sum_{j=1}^{N} V S I_{i j}
$$

$V S I_{i}$ can be estimated for every bus and the buses with higher values are selected as the candidate buses to add dynamic var sources.

\section{B. Optimize the Sizes of Dynamic Var Sources}

Assume that there are $n$ candidate buses, i.e. $\left|I_{Q}\right|=n$. The heuristic approach proposed in this paper conducts the following steps to determine the sizes of the dynamic var sources (taking SVCs as an example), i.e. $Q_{1} \sim Q_{n}$ : 
1. In the $n$-dimensional space of $Q_{1} \sim Q_{n}$, the initial guesses (denoted by $Q_{1}{ }^{0} \sim Q_{n}{ }^{0}$ ) are selected at a feasible solution meeting all post-fault voltage criteria or at a solution without violating voltage criterion (7). Let $k=0$

2. Time-domain simulation on the most severe contingency is conducted with SVC models of sizes $Q_{1}{ }^{k} \sim Q_{n}{ }^{k}$ added to the candidate buses. Then, add a small amount $q_{i}$, e.g. $Q_{i}{ }^{k} / 10$, to each candidate bus and re-simulate the contingency. Calculate $V S I_{i j}$ using the voltage responses with and without $q_{i}$ by (9).

3. Use $V S I_{i j}$ as heuristic information to optimize the amount of update $\Delta Q_{i}{ }^{k}$ by solving a LP problem. Then, let $Q_{i}{ }^{k+1}=Q_{i}{ }^{k}+\Delta Q_{i}{ }^{k}$

4. Iterate Steps 2 and 3 until one of the following stopping criteria is met:

i. If all $\left|\Delta Q_{i}{ }^{k}\right|$ 's are smaller than an inaccuracy limit $\mathcal{E}$ and the voltage responses with $Q_{i}{ }^{k+1}$ satisfy all voltage criteria, then let $Q_{1} \sim Q_{n}=Q_{1}^{k+1} \sim Q_{n}{ }^{k+1}$

ii. If $k+1$ exceeds a preset maximum iteration number $K$ (e.g. 10 20), let $Q_{1} \sim Q_{n}$ take the results from the most recent iteration that does not cause any violation of voltage criteria

The second stopping criterion is to make sure the feasibility of the final solution in case that the LP solution after a number of iterations violates voltage criterion (7). If then, the most recent feasible solution is provided, instead. Detailed algorithms in Step 3 to determine $\Delta Q_{1}^{k} \sim \Delta Q_{n}^{k}$ are presented below.

As illustrated in Fig. 1, define

$$
\begin{gathered}
\Delta V_{j}^{L, k}=\min \left[V_{j}^{k, t}, t=1 \sim T\right]-V_{j}^{L} \\
\Delta V_{j}^{U, k}=V_{j}^{U}-\max \left[V_{j}^{k, t}, t=1 \sim T\right]
\end{gathered}
$$

where $V_{j}^{k, t}$ is the voltage of bus $j$ at time step $t$, and $V_{j}^{L}$ $=0.75 V_{j}^{i \text { init }}$ if $t<t_{s}$ or $0.95 V_{j}^{\text {init }}$, otherwise, and $V_{j}^{U}=1.25 V_{j}^{\text {init }}$ if $t<t_{s}$ or $1.05 V_{j}^{\text {init }}$, otherwise. The magnitudes of $\Delta V_{j}^{L, k}$ and $\Delta V_{j}^{U, k}$ are actually the closest distances from the voltage trajectory to the two limits. $\Delta V_{j}^{L, k}\left(\Delta V_{j}^{U, k}\right)$ is positive if the trajectory is above (below) the limit, or negative, otherwise.

Then the following LP problem is solved to determine $\Delta Q_{1}^{k} \sim \Delta Q_{n}^{k}$.

Minimize

$$
f=\sum_{i=1}^{n} c_{i} Q_{i}^{k+1}
$$

Subject to

$$
\begin{gathered}
Q_{i}^{k+1}=Q_{i}^{k}+\Delta Q_{i}^{k}, \forall i=1 \sim n \\
-\sum_{i=1}^{n} \alpha_{i j} V S I_{i j} \Delta Q_{i}^{k} \leq \Delta V_{j}^{L, k}, \forall j=1 \sim N \\
\sum_{i=1}^{n} \beta_{i j} V S I_{i j} \Delta Q_{i}^{k} \leq \Delta V_{j}^{U, k}, \forall j=1 \sim N
\end{gathered}
$$

where $\alpha_{i j}$ and $\beta_{i j}$ are an acceleration factors with a default value 1 . The above LP problem is to approximately find the minimum var requirement to improve post-fault voltage performance by pushing the post-fault voltage trajectories as close to the lower voltage limit as possible. $\Delta Q_{1}^{k} \sim \Delta Q_{n}^{k}$ are solved to update $Q_{1}^{k} \sim Q_{n}^{k}$ for the next iteration. The constraints (14) and (15) are because the voltage response is expected to stay within the two limits and approach the lower limit to save the total cost.

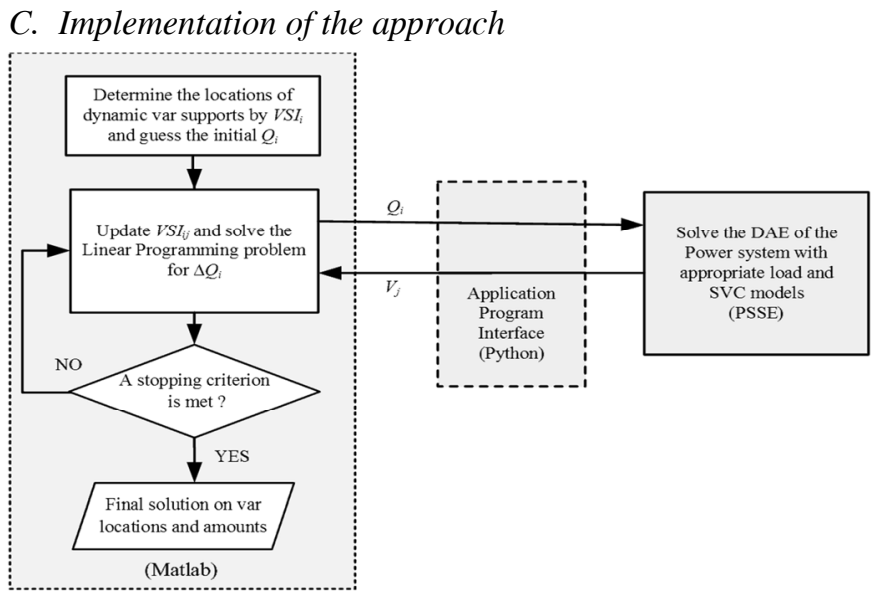

Figure 2. Flow chart for implementing the proposed approach

Fig. 2 illustrates the implementation of the new approach. The approach interfaces the LP algorithm (MATLAB code) with the power system dynamics simulator (i.e. PSS/E) via Python. PSS/E solves the DAEs in (8) to simulate the postfault voltage responses for any given var injection strategy. The results are fed back to the LP algorithm to check voltage criteria and calculate $V S I_{i j}, \Delta V_{j}^{L, k}$ and $\Delta V_{j}^{U, k}$. Induction motor loads and SVCs needed to be modeled in PSS/E simulations.

\section{CASE STUdies}

The proposed new approach is tested by case studies on the WSCC 9-bus system and IEEE 39-bus system. The studies apply the CLODBL load model and CSVGN5 SVC model (see [9] for details) to simulate the phenomena of FIDVR and dynamic var supporting. In the case studies, we assume all $c_{i}=1$, i.e. all buses have identical costs for SVCs. In this case the objective is actually to minimize the total amount of dynamic var supports to meet the voltage criteria. The total post-fault simulation time is $5 \mathrm{~s}$ and the post transient criterion is checked after the first 3 seconds transient period. No upper limit is set for the size of dynamic var supports. For both case studies, let $q_{i}=5 \mathrm{Mvar}$, the maximum iteration number $K=10$, and inaccuracy limit $\varepsilon=1$ Mvar.

\section{A. 9-bus system}

The 9-bus system has 3 generators, 3 transformer, 3 loads, and 5 transmission lines, as shown in Fig. 3. According to contingency analysis, a three-phase fault on bus 7 , which is cleared by opening the line between buses 7-5 after 5 cycles, is identified as the most severe $\mathrm{N}-1$ contingency. The delayed voltage recovery phenomenon is simulated as shown in Fig.4. 5 out of 9 bus voltages violate the voltage criteria. 
At Buses 6 and 8, the overall VSI is higher than that at other buses. So they are selected as two candidate buses to install SVCs. Assume the sizes of the two SVCs are larger than 1 Mvar. The initial guesses of $Q_{6}$ and $Q_{8}$ are set at $30 \mathrm{Mvar}$ and 50Mvar, respectively. As shown in Fig. 5, after 4 iterations the proposed approach converges to a solution: $Q_{6}=56 \mathrm{Mvar}$ and $Q_{8}=1 \mathrm{Mvar}$. To investigate the global optimality of this solution, an exhaustive search is conducted to identify the entire region of feasible solutions as shown by the gray region in Fig. 5. It is confirmed that the obtained solution is the global optimum although the solution region is non-convex. For this case, the initial guesses do not correspond to a feasible solution but the first iteration jumps to a feasible solution. To test the voltage performance with the optimal var injections, time-domain simulation is conducted with SVCs added to Buses 6 and 8 with the determined sizes. Fig. 6 shows that all trajectories meet the voltage criteria.

The approach in [6] is implemented with 0.005 s time step to compare with the new approach. It is found that the two approaches give identical solutions. However, the new approach is about 10 times faster for this case using an Intel i5-3220M computer. The reason is because the new approach only handles a LP problem and it does not need to interface the optimization solver with power system simulator for each time step as the approach in [6] does.

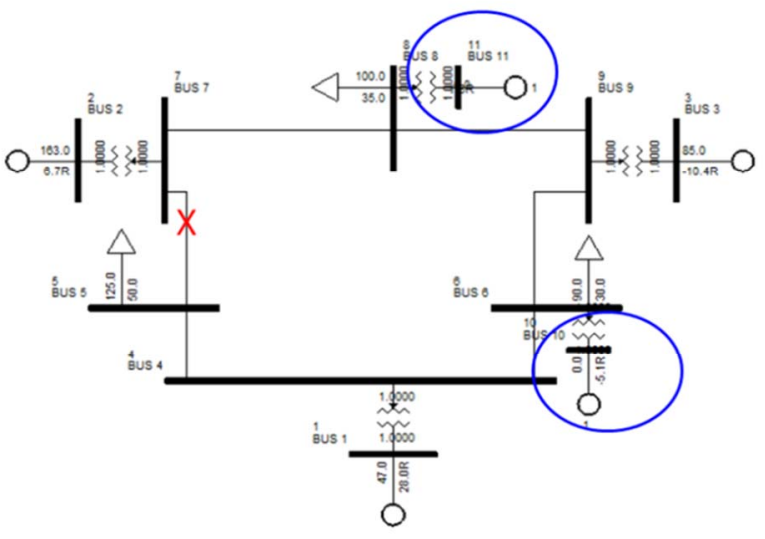

Figure 3. 9-bus system with the most severe $\mathrm{N}-1$ contingency and two candidate buses for dynamic var supports

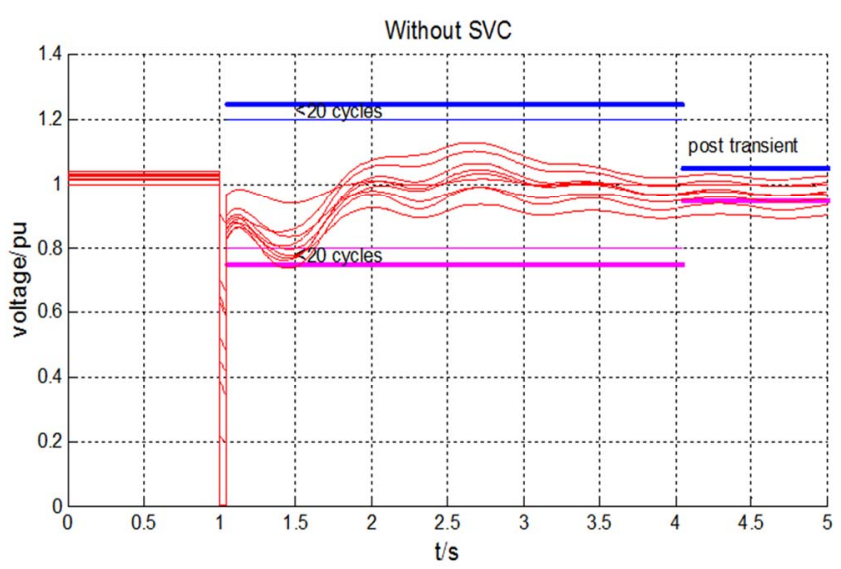

Figure 4. Bus voltage responses of the 9-bus system

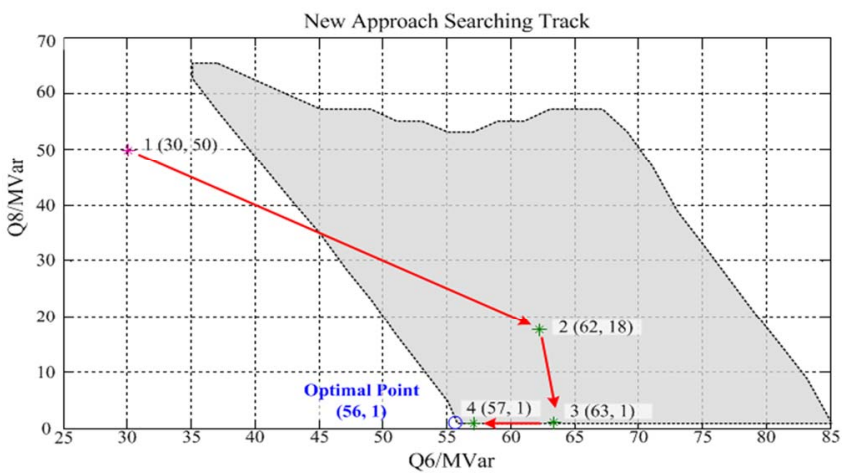

Figure 5. Searching path of the new approach for the 9-bus system

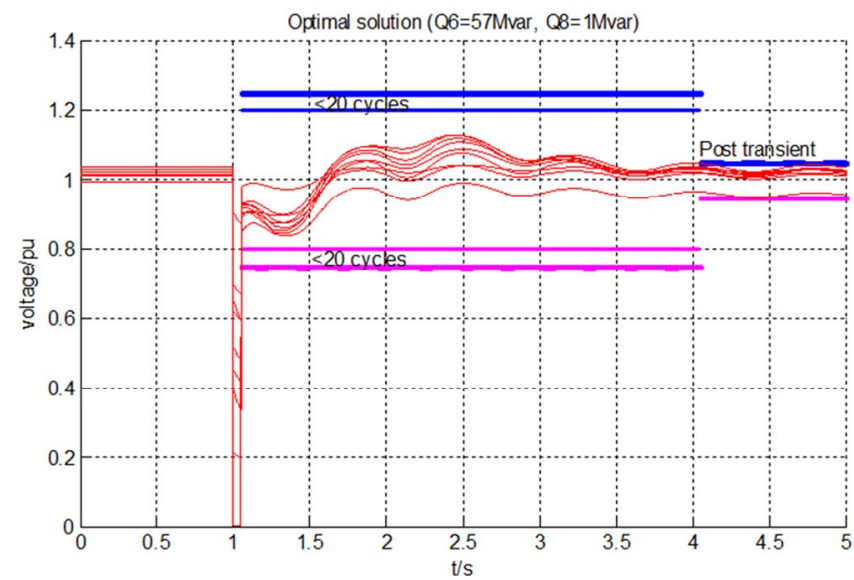

Figure 6. Post-fault voltage responses with optimized SVCs for the 9-bus system

\section{B. IEEE 39-bus System}

As shown in Fig. 7, the IEEE 39-bus system is also used to test the proposed approach. The most severe contingency is identified as a three phase fault on Bus 17 cleared by opening Lines 17-18 and 17-27 after 5 cycles. Post-fault voltage trajectories are shown in Fig. 8. Some buses violate the $25 \%$ voltage deviation limit at the beginning of the 3-second transient period or the 5\% limit at the beginning of the post transient period.

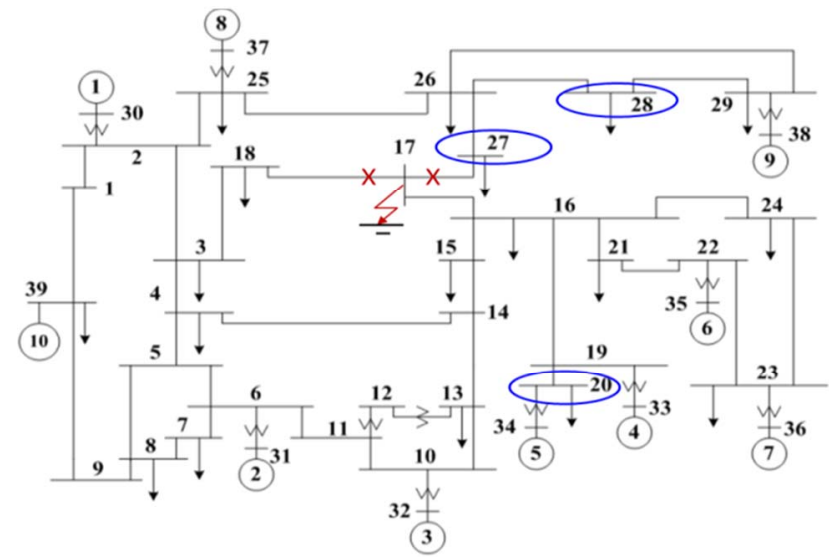

Figure 7. IEEE 39-bus system and the most severe $\mathrm{N}-2$ contingency and three candidate buses for dynamic var supports 
Assume that the sizes of SVCs are larger than 25Mvar and have no upper limits. The new approach is applied to find the optimal locations and sizes of SVCs to support the voltage recovery. First, Buses 20, 27, and 28 are found to have the highest VSI values and thus selected to be candidate buses. Then, conduct the heuristic searching algorithm to optimize $Q_{20}, Q_{27}$ and $Q_{28}$. Select an initial point $(280,280,280)$ in the space of $Q_{20}, Q_{27}$ and $Q_{28}$. As shown in Fig. 9, after 4 iterations the optimal solution is found to be $Q_{20}=326$ Mvar and $Q_{27}=Q_{28}=25$ Mvar. This solution is verified to be the global optimum by an exhaustive search in the 3-dimensional space. Fig. 9 also shows the boundary of feasible solutions by the gray plane.

The optimal solution is tested by time-domain simulation with SVCs of the optimized sizes added at the three candidate buses. Fig. 10 shows that all post-fault voltage trajectories meet the voltage criteria.

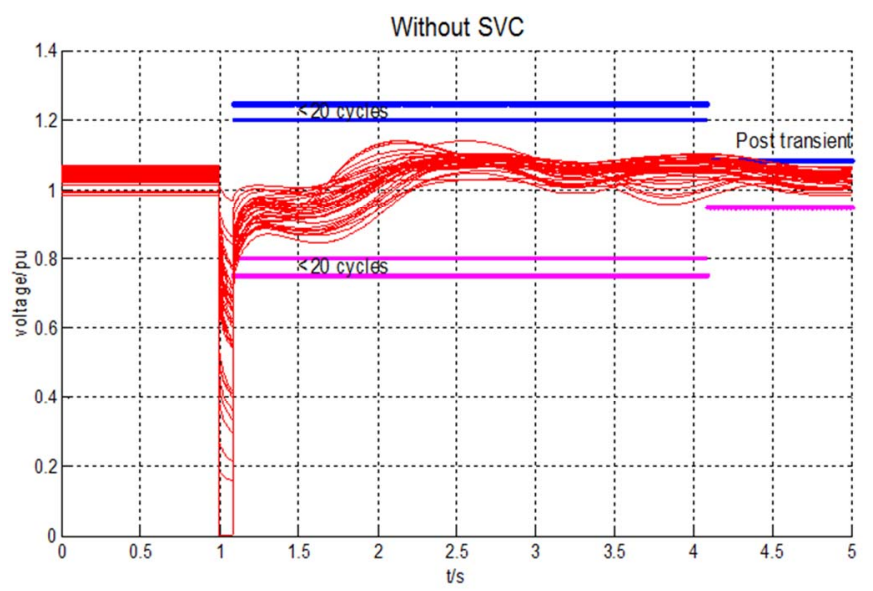

Figure 8. Post-fault voltage responses of the IEEE 39-bus system

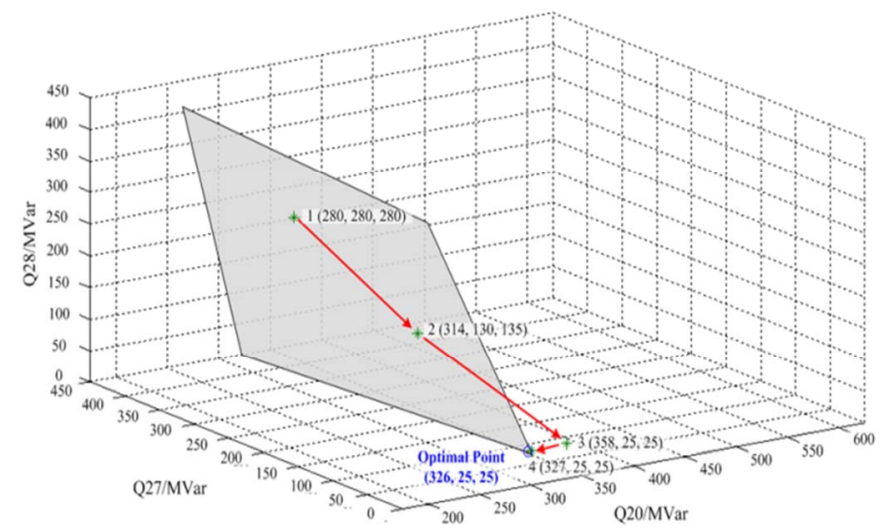

Figure 9. Searching path of the new approach for the IEEE 39-bus system

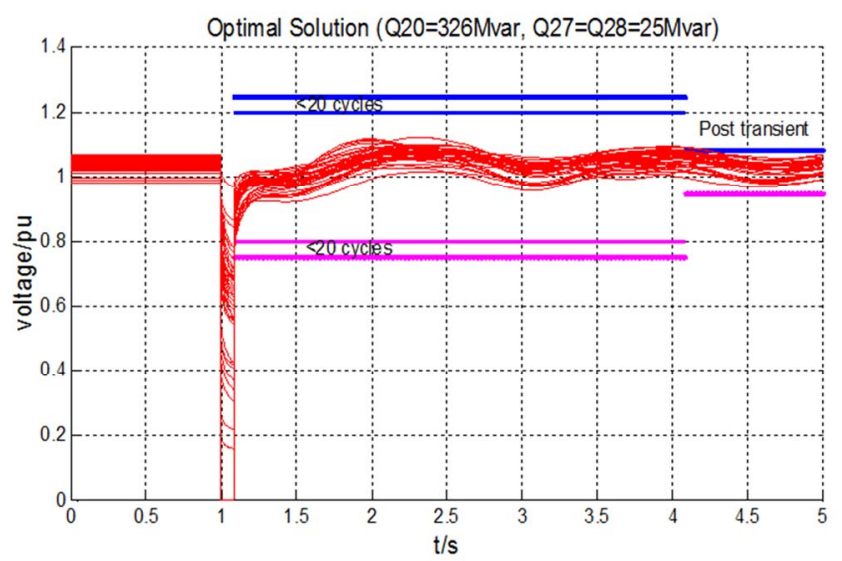

Figure 10. Post-fault voltage responses with optimized SVCs of the IEEE 39-bus system

\section{CONCLUSION}

This paper proposes a new approach to optimal allocation of dynamic var sources to address the FIDVR problem. The new approach was benchmarked with the approach in reference [6] and it is more efficient thanks to its heuristic searching algorithm. Future work will include validation of the new approach on realistic power system models.

\section{REFERENCES}

[1]. B. Sapkota and V. Vittal, "Dynamic var planning in a large power system using trajectory sensitivities," IEEE Trans. Power Syst., vol. 25, no. 1, pp. 461-469, Feb. 2010.

[2]. P. Pourbeik, R. J. Koessler, W. Quaintance, and W. Wong, "Performing comprehensive voltage stability studies for the determination of optimal location, size and type of reactive compensation," IEEE PES General Meeting. 2006.

[3]. V.S. Kolluri, and S. Mandal, "Determining reactive power requirements in the southern part of the energy system for improving voltage security-A case study", IEEE PSCE, 2006.

[4]. A. P. S. Meliopoulos, G. Cokkinides, and G. Stefopoulos, "Voltage stability and voltage recovery: load dynamics and dynamic var sources", IEEE PES General Meeting, Oct. 2006.

[5]. A. Tiwari and V. Ajjarapu, "Optimal allocation of dynamic var support using mixed integer dynamic optimization", IEEE Trans. Power Syst., vol. 26, no. 1, pp. 305-314, Feb. 2011.

[6]. M. Paramasivam, et al, , "Dynamic optimization based reactive power planning to mitigate slow voltage recovery and short term voltage instability", IEEE Trans. Power Syst., vol. 28, no. 4, pp. 3865-3873, Nov. 2013.

[7]. D. J. Shoup, J. J. Paserba, and C. W. Taylor, "A survey of current practices for transient voltage dip/sag criteria related to power system stability," IEEE PES PSCE. Oct, 2004.

[8]. NERC/WECC Planning Standards, WECC, Apr., 2003.

[9]. Siemens PTI Power Technologies Inc., PSS/E 33, Program Application Guide, vol. II, May 2011. 